\title{
Heparan sulfate provides a mechanism to respond to FGFR2b and control regenerative expansion
}

\author{
Enrique Brandan
}

Received: 18 January 2015 / Accepted: 18 January 2015 / Published online: 26 February 2015

(C) The International CCN Society 2015

\begin{abstract}
Fast varying 3- $O$-sulfated groups in epithelial KIT+ progenitors provides a cellular mechanism to respond to FGFR2b-mediated signaling and expansion of fetal and adult progenitors. This is allowed by the synthesis of 3-O-sulfated heparan sulfate that up-regulate KIT and 3-O-sulfotransferase enzymes, augmenting 3-O-sulfated heparan sulfate.
\end{abstract}

Keywords Heparan sulfate proteoglycans · Signaling · FGFR2b - KIT

Heparan sulfate proteoglycans has gained a lot of attention for the modulation of growth factor activities among other functions. Patel et al. (2014), show in KIT+ progenitors cells from fetal salivary glands under expansive progenitor expansion, that 3-O sulfotransferases (3-O-HS) (enzymes responsible to modify heparan sulfate proteoglycans) are enriched and its expression is directly regulated by the FGF-10/FGFR2b. Autocrine feedback increases 3-O-HS, KIT, and progenitor expansion. Knockdown of multiple $H s 3 s t$ isoforms limit fetal progenitor expansion but is rescued by bioengineered 3-OHS. These results indicate that altering 3-O sulfated groups in heparan sulfate provides a mechanism to respond to FGFR2b and control regenerative expansion.

This work elegantly shows that heparan sulfate chain modifications are finely regulated and in this case in an autocrine fashion. If we think that there on average 4 different sulfate groups per disaccharide of heparan sulfate and seven 3-OH-HS just for 3-O-sulfation, the possibilities to regulate structure and therefore function of heparan sulfate proteoglycans are enormous and unexpected. The same might be true to other enzymes involved in heparan sulfate modifications.

\section{Reference}

Patel VN, Lombaert IM, Cowherd SN, Shworak NW, Xu Y, Liu J, Hoffman MP (2014) Dev Cell 29(6):662-73. doi:10.1016/j.devcel. 2014.04.024
E. Brandan $(\bowtie)$

Pontificia Universidad Católica de Chile, Santiago, Chile

e-mail: ebrandan@bio.puc.cl 